\title{
The existence of strong complete mappings
}

\author{
Anthony B. Evans \\ Department of Mathematics and Statistics \\ Wright State University \\ Dayton, Ohio 45435 \\ anthony. evans@wright.edu
}

Submitted: Feb 9, 2011; Accepted: Jan 23, 2012; Published: Feb 7, 2012

Mathematics Subject Classification: 05B15

\begin{abstract}
A strong complete mapping of a group $G$ is a bijection $\theta: G \rightarrow G$ for which both mappings $x \mapsto x \theta(x)$ and $x \mapsto x^{-1} \theta(x)$ are bijections. We characterize finite abelian groups that admit strong complete mappings, thus solving a problem posed by Horton in 1990. We also prove the existence of strong complete mappings for countably infinite groups.
\end{abstract}

\section{Introduction}

For a group $G$, a bijection $\theta: G \rightarrow G$ is a complete mapping of $G$ if the mapping $x \mapsto x \theta(x)$ is a bijection, an orthomorphism of $G$ if the mapping $x \mapsto x^{-1} \theta(x)$ is a bijection, and a strong complete mapping of $G$ if it is both a complete mapping of $G$ and an orthomorphism of $G$. The term strong complete mapping was first used by Hsu and Keedwell [10] in 1985: these were called strong orthomorphisms in [1], and strong permutations in [9].

Which groups admit strong complete mappings? To admit strong complete mappings, a group must first admit complete mappings. For infinite groups the existence problem for complete mappings was settled in 1950 by Bateman [2], who proved that any infinite group admits complete mappings. In 1955 Hall and Paige [6] proved that a finite group with a nontrivial, cyclic Sylow 2-subgroup does not admit complete mappings: the converse was proved in 2009 by Wilcox [14], Evans [5], and Bray [3]. Thus the existence problem for complete mappings is settled. For strong complete mappings, however, the existence problem is far from settled.

In 1947 Paige [12] characterized finite abelian groups that admit complete mappings. A finite abelian group admits complete mappings if and only if its Sylow 2-subgroup is either trivial or noncyclic. In determining whether a finite abelian group admits strong complete mappings or not we need to consider, not only the structure of its Sylow 2subgroup, but also the structure of its Sylow 3-subgroup. For cyclic groups, the existence 
problem for strong complete mappings was implicitly settled by Hedayat and Federer in the context of Knut Vic designs: a Knut Vic design of order $n$ exists if and only if $\mathbb{Z}_{n}$ admits strong complete mappings. In 1975 Hedayat and Federer [8] proved that a Knut Vic design of order $n$ exists if neither 2 nor 3 divides $n$, and Hedayat [7] proved the converse in 1977. For only a few other classes of finite groups has the existence problem for strong complete mappings been settled. As an example: in 1990 Evans [4] and Horton [9] independently proved that noncyclic abelian 2-groups admit strong complete mappings. In 1990 Horton [9] posed the problem of characterizing finite abelian groups that admit strong complete mappings. We will solve this problem by proving that a finite abelian group admits strong complete mappings if and only if neither its Sylow 2-subgroup nor its Sylow 3-subgroup is nontrivial and cyclic.

In Section 2 we will survey known nonexistence results. In Section 3 we will characterize finite abelian groups that admit strong complete mappings, and in Section 4 we will prove that all countably infinite groups, whether abelian or not, admit strong complete mappings.

\section{Nonexistence results}

For a group to admit strong complete mappings it must first admit complete mappings. In 1955 Hall and Paige [6] proved that a necessary condition for a finite group to admit complete mappings was that its Sylow 2-subgroup be either trivial or noncyclic.

Theorem 1 (Hall, Paige, 1955). A finite group with a nontrivial, cyclic Sylow 2-subgroup does not admit complete mappings.

Proof. See Theorem 5 in [6].

Hall and Paige conjectured the converse of Theorem 1. This conjecture was proved in 2009: Wilcox [14] proved that any minimal counterexample to the Hall-Paige conjecture must be a nonabelian simple group and further showed that it would have to be the Tits group or a sporadic simple group; Evans [5] reduced the number of possible minimal counterexamples to just one, $J_{4}$, Janko's fourth group; and Bray [3] completed the proof by showing that $J_{4}$ could not be a minimal counterexample.

Theorem 2 (Wilcox, Evans, Bray, 2009). A finite group with a trivial or noncyclic Sylow 2-subgroup admits complete mappings.

In 1990 Evans [4] established a similar necessary condition for a finite group to admit strong complete mappings.

Theorem 3 (Evans, 1990). If a finite group $G$ has a nontrivial, cyclic Sylow 3-subgroup $S$ and $H$ is a normal subgroup of $G$ for which $G / H \cong S$, then $G$ does not admit strong complete mappings.

Proof. See Theorem 2 in [4]. 
This result can be strengthened for groups of odd order.

Corollary 4. A finite group of odd order with a nontrivial, cyclic Sylow 3-subgroup does not admit strong complete mappings.

Proof. If $G$ is of odd order and has a cyclic Sylow 3-subgroup $H$, then there is a homomorphism from $G$ onto $H$ : see Corollary 1.4 .18 in [11] for a proof. The result then follows from Theorem 3.

Horton [9] proved Corollary 4 for abelian groups. It should be noted that Horton's proof, Evans' proof of Theorem 3, and Hedayat's [7] 1977 proof that the order of a Knut Vic design cannot be divisible by 3 , are essentially the same.

Analogous to the Hall-Paige conjecture, we conjecture a partial converse to Theorem 3.

Conjecture 5. A finite group whose Sylow 2-subgroup is trivial or noncyclic and whose Sylow 3-subgroup is also trivial or noncyclic admits strong complete mappings.

If the Sylow 3-subgroup of a finite group is nontrivial and cyclic, but not a homomorphic image, is it still possible for the group to admit strong complete mappings? Of necessity, if such a group admits strong complete mappings, then it must be of even order and its Sylow 2-subgroup must be noncyclic. The smallest case of interest is $D_{6}$, the dihedral group of order 12: Shieh, Hsiang, and Hsu [13] have shown that this group admits strong complete mappings.

\section{Abelian groups}

In 1990 Horton [9] posed the problem of determining which finite abelian groups admit strong complete mappings. Also in 1990, Evans [4] conjectured that these groups would be precisely those with a trivial or noncyclic Sylow 2-subgroup and a trivial or noncyclic Sylow 3-subgroup. In this section we will prove this conjecture true. In Lemmas 6 through 9 we list some known existence results for strong complete mappings.

Lemma 6. Any group whose order is divisible by neither 2 nor 3 admits strong complete mappings.

Proof. Let $G$ be a group of order $n$. If $\operatorname{gcd}(n, r)=1$, then the mapping $x \mapsto x^{r}$ is a bijection. It follows that, if $n$ is divisible by neither 2 nor 3 , then $x \mapsto x^{2}$ is a strong complete mapping.

Lemma 7. If $q \geqslant 4$, then the elementary abelian group $G F(q)^{+}$admits strong complete mappings.

Proof. The mapping $x \mapsto a x$ is a strong complete mapping if $a \neq 0, \pm 1$.

Lemma 8. If $H$ is a subgroup of an abelian group $G$ and $G / H$ and $H$ both admit strong complete mappings, then $G$ admits strong complete mappings. 
Proof. See Theorem 3 in [4] or Lemma 2.8 in [9].

Recently Shieh, Hsiang, and Hsu [13] have proved that Lemma 8 holds for nonabelian groups also.

Lemma 9. Every noncyclic, abelian 2-group admits strong complete mappings.

Proof. See Theorem 4 in [4] or Lemma 2.10 in [9].

To prove that noncyclic, abelian 3-groups admit strong complete mappings we will need to prove the existence of strong complete map pings for 3-groups of the form $G=\mathbb{Z}_{3} \times \mathbb{Z}_{3 m}$. For the two smallest cases, $\mathbb{Z}_{3} \times \mathbb{Z}_{3}$ admits strong complete mappings by Lemma 7 , and in 1990 Horton [9] found a strong complete mapping of $\mathbb{Z}_{3} \times \mathbb{Z}_{9}$ via a computer search.

Let $G=\mathbb{Z}_{3} \times \mathbb{Z}_{3 m}, m \geqslant 3$ a power of 3 , and let $K$ be the subgroup of $G$ generated by $(1,0)$ and $(0, m)$. Thus $K$ is isomorphic to the vector space of dimension 2 over $G F(3)$. We will use $i j$ to denote the element $(i, j m)$ of $K$ and, if $k=i j \in K$ and $l \in\{0, \ldots, m-1\}$, we will use $[k, l]$ or $[i j, l]$ to denote the element $(i, j m+l)$ of $G$. For $i j \in K$ we will call $i$ the first component and $j$ the second component of $i j$.

For $i=0,1,2$ and $j=0,1, \ldots, m-1$, let $\delta_{i, j}, \epsilon_{i, j} \in K$ be defined by

$$
\delta_{i, j}= \begin{cases}01 & \text { if }(3 j+i \quad \bmod m)<j \\ 00 & \text { otherwise }\end{cases}
$$

and

$$
\epsilon_{i, j}= \begin{cases}01 & \text { if }(3 j+i \quad \bmod m) \geqslant m-j, \\ 00 & \text { otherwise }\end{cases}
$$

For $a \in\{1, \ldots, m-1\}, \operatorname{gcd}(a, m)=1$, let $a^{\prime}$ denote the unique integer in $\{1, \ldots, m-1\}$ satisfying $a a^{\prime} \equiv 1(\bmod m)$.

Lemma 10. $\delta_{i, j}=01$ if and only if $j \in\{m / 3, \ldots,(m-3) / 2\} \cup\{2 m / 3, \ldots, m-2\}$ and $i=0,1,2$, or $j=(m-1) / 2$ and $i=0$, or $j=m-1$ and $i=0,1$.

Proof. By definition $\delta_{i, j}=01$ if and only if $(3 j+i \bmod m)<j$, if and only if $0 \leqslant$ $3 j+i-k m<j$ for some integer $k$. As $0 \leqslant j \leqslant m-1$, this inequality can hold true for $k=1$ or 2 only. If $k=1$, then the inequality holds if and only if

$$
\frac{m}{3} \leqslant j \leqslant \begin{cases}\frac{m-1}{2} & \text { if } i=0, \\ \frac{m-3}{2} & \text { if } i=1,2 .\end{cases}
$$

If $k=2$, then the inequality holds if and only if

$$
\frac{2 m}{3} \leqslant j \leqslant \begin{cases}m-1 & \text { if } i=0,1 \\ m-2 & \text { if } i=2 .\end{cases}
$$

Hence the result. 
The following lemma gives a sufficient condition for $\mathbb{Z}_{3} \times \mathbb{Z}_{3 m}, m$ a power of 3 , to admit strong complete mappings.

Lemma 11. The group $G=\mathbb{Z}_{3} \times \mathbb{Z}_{3 m}, m \geqslant 3$ a power of 3 , admits strong complete mappings if there exist $\alpha_{i, j} \in K, i=0,1,2$ and $j=0,1, \ldots, m-1$, and $x_{j} \in\{01,02\}$, $j=0,1, \ldots, m-1$, for which the following three conditions hold.

1. For $i=0,1,2$ and $j=0,1, \ldots, \frac{m}{3}-1$, the set

$$
\left\{\alpha_{i, j}, \alpha_{i, j+m / 3}, \alpha_{i, j+2 m / 3}\right\}
$$

is a system of distinct coset representatives for $\langle 11\rangle$ in $K$.

2. For $j=0,1, \ldots, m-1$, the set

$$
\left\{\alpha_{0, j}-\delta_{0, j}, \alpha_{1, j-2^{\prime}}-x_{j-2^{\prime}}-\delta_{1, j-2^{\prime}}, \alpha_{2, j-1}+x_{j-1}-\delta_{2, j-1}\right\}
$$

is a system of distinct coset representatives for $\langle 10\rangle$ in $K$, where the indices are computed modulo m.

3. For $j=0,1, \ldots, m-1$, the set

$$
\left\{\alpha_{0, j}, \alpha_{1, j-4^{\prime}}, \alpha_{2, j-2^{\prime}}\right\}
$$

is a system of distinct coset representatives for $\langle 01\rangle$ in $K$, where the second indices are computed modulo m.

Proof. Let $H$ be the subgroup of $K$ generated by 12 and let $A=\left(\begin{array}{ll}0 & 0 \\ 1 & 1\end{array}\right)$. Then $H A=\langle 11\rangle, H(A-I)=\langle 10\rangle$, and $H(A+I)=\langle 01\rangle$. Let $\alpha_{i, j} \in K, i=0,1,2$ and $j=0,1, \ldots, m-1$, and $x_{j} \in\{01,02\}, j=0,1, \ldots, m-1$, satisfy the conditions of the lemma and let $\theta: G \rightarrow G$ be define d by

$$
\theta\left(\left[h+i x_{j}, j\right]\right)=\left[h A+\alpha_{i, j},(3 j+i \bmod m)\right],
$$

where $h \in H, i \in\{0,1,2\}$, and $j \in\{0,1, \ldots, m-1\}$.

Clearly $\theta$ is well-defined.

As

$$
3 j+i \equiv 3\left(j+\frac{m}{3}\right)+i \equiv 3\left(j+\frac{2 m}{3}\right)+i \quad(\bmod m),
$$

$\theta$ is a bijection if and only if, the set $\left\{\alpha_{i, j}, \alpha_{i, j+m / 3}, \alpha_{i, j+2 m / 3}\right\}, i=0,1,2$ and $j=$ $0,1, \ldots, \frac{m}{3}-1$, is a system of distinct coset representatives for $\langle 11\rangle$ in $K$.

Now $\theta\left(\left[h+i x_{j}, j\right]\right)-\left[h+i x_{j}, j\right]=\left[h(A-I)-i x_{j}+\alpha_{i, j}-\delta_{i, j},(2 j+i \bmod m)\right]$. As $2 j \equiv$ $2\left(j-2^{\prime}\right)+1 \equiv 2(j-1)+2(\bmod m)$, the mapping $\left[h+i x_{j}, j\right] \mapsto \theta\left(\left[h+i x_{j}, j\right]\right)-\left[h+i x_{j}, j\right]$ is a bijection if and only if, for $j=0,1, \ldots, m-1$, the set $\left\{\alpha_{0, j}-\delta_{0, j}, \alpha_{1, j-2^{\prime}}-x_{j-2^{\prime}}-\right.$ $\left.\delta_{1, j-2^{\prime}}, \alpha_{2, j-1}+x_{j-1}-\delta_{2, j-1}\right\}$ is a system of distinct coset representatives for $\langle 10\rangle$ in $K$. 
Now $\theta\left(\left[h+i x_{j}, j\right]\right)+\left[h+i x_{j}, j\right]=\left[h(A+I)+i x_{j}+\alpha_{i, j}+\epsilon_{i, j},(4 j+i \bmod m)\right]$. As $4 j \equiv 4\left(j-4^{\prime}\right)+1 \equiv 4\left(j-2^{\prime}\right)+2(\bmod m)$ and $x_{j}, \epsilon_{i, j} \in H(A+I)$ for all $i, j$, the mapping $\left[h+i x_{j}, j\right] \mapsto \theta\left(\left[h+i x_{j}, j\right]\right)+\left[h+i x_{j}, j\right]$ is a bijection if and only if, for $j=0,1, \ldots, m-1$, the set $\left\{\alpha_{0, j}, \alpha_{1, j-4^{\prime}}, \alpha_{2, j-2^{\prime}}\right\}$ is a system of distinct coset representatives for $\langle 01\rangle$ in $K$. The result follows.

We call the condition in Lemma 11(1) the $\Theta$ condition, the condition in Lemma 11(2) the $\Delta$ condition, and the condition in Lemma 11(3) the $\Sigma$ condition. We call the sets $\left\{\alpha_{i, j}, \alpha_{i, j+m / 3}, \alpha_{i, j+2 m / 3}\right\}, \Theta$ sets; the sets $\left\{\alpha_{0, j}-\delta_{0, j}, \alpha_{1, j-2^{\prime}}-x_{j-2^{\prime}}-\delta_{1, j-2^{\prime}}, \alpha_{2, j-1}+\right.$ $\left.x_{j-1}-\delta_{2, j-1}\right\}, \Delta$ sets; and the sets $\left\{\alpha_{0, j}, \alpha_{1, j-4^{\prime}}, \alpha_{2, j-2^{\prime}}\right\}, \Sigma$ sets. The differences, first components minus second components modulo $m$, will be called the component differences. The $\Theta$ condition is satisfied if and only if, for each $\Theta$ set, the component differences are all distinct. The $\Delta$ condition is satisfied if and only if, for each $\Delta$ set, the second components are all distinct, and the $\Sigma$ condition is satisfied if and only if, for each $\Sigma$ set, the first components are all distinct.

As a corollary to Lemma 11 , we can show that $\mathbb{Z}_{3} \times \mathbb{Z}_{9}$ and $\mathbb{Z}_{3} \times \mathbb{Z}_{27}$ admit strong complete mappings.

Corollary 12. The groups $\mathbb{Z}_{3} \times \mathbb{Z}_{9}$ and $\mathbb{Z}_{3} \times \mathbb{Z}_{27}$ admit strong complete mappings.

Proof. For $m=3$, let $x_{0}=01, x_{1}=02, x_{2}=01$, and, for $i, j=0,1,2$, let $\alpha_{i, j}$ be the $i j$ th entry in the following table.

\begin{tabular}{c|ccc} 
& 0 & 1 & 2 \\
\hline 0 & 00 & 01 & 02 \\
1 & 11 & 20 & 10 \\
2 & 11 & 20 & 21
\end{tabular}

It is routine to show that the conditions of Lemma 11 are satisfied and, hence, that $\mathbb{Z}_{3} \times \mathbb{Z}_{9}$ admits strong complete mappings. The corresponding strong complete mapping is described in the table below.

\begin{tabular}{c|c||c|c||c|c||c|c||c|c||c|c}
$x$ & $\theta(x)$ & $x$ & $\theta(x)$ & $x$ & $\theta(x)$ & $x$ & $\theta(x)$ & $x$ & $\theta(x)$ & $x$ & $\theta(x)$ \\
\hline 00 & 00 & 26 & 27 & 17 & 20 & 04 & 22 & 25 & 10 & 15 & 12 \\
16 & 26 & 06 & 15 & 24 & 16 & 11 & 18 & 05 & 11 & 22 & 08 \\
23 & 13 & 13 & 02 & 07 & 21 & 27 & 05 & 12 & 07 & & \\
03 & 14 & 20 & 28 & 14 & 17 & 02 & 06 & 28 & 24 & & \\
10 & 01 & 01 & 03 & 21 & 04 & 18 & 23 & 08 & 25 & &
\end{tabular}

For $m=9$, let

$$
x_{j}= \begin{cases}01 & \text { if } j=3,4,5 \\ 02 & \text { otherwise }\end{cases}
$$

and, for $i=0,1,2$ and $j=0,1, \ldots, 8$, let $\alpha_{i, j}$ be the $i j$ th entry in the following table.

\begin{tabular}{c|ccccccccc} 
& 0 & 1 & 2 & 3 & 4 & 5 & 6 & 7 & 8 \\
\hline 0 & 00 & 12 & 00 & 10 & 00 & 12 & 20 & 10 & 10 \\
1 & 02 & 22 & 12 & 20 & 12 & 21 & 11 & 21 & 11 \\
2 & 02 & 00 & 21 & 00 & 20 & 00 & 20 & 02 & 20
\end{tabular}


It is routine to show that the conditions of Lemma 11 are satisfied and, hence, that $\mathbb{Z}_{3} \times \mathbb{Z}_{27}$ admits strong complete mappings.

More generally, $\mathbb{Z}_{3} \times \mathbb{Z}_{3 m}$ admits strong complete mappings.

Lemma 13. If $m=3^{n}, n \geqslant 2$, then $\mathbb{Z}_{3} \times \mathbb{Z}_{3 m}$ admits strong complete mappings.

Proof. We will choose $\alpha_{i, j}$ and $x_{j}, i=0,1,2$ and $j=0, \ldots, m-1$, so that the conditions of Lemma 11 are satisfied.

We set

$$
x_{j}= \begin{cases}01 & \text { if } j=2 m / 3, \ldots,(5 m-9) / 6 \\ 02 & \text { otherwise }\end{cases}
$$

$\alpha_{0, j}=0 k$, where

$$
k= \begin{cases}0 & \text { if } 0<j<m / 3, \text { or } j=2 m / 3 \\ 1 & \text { if } m / 3 \leqslant j<2 m / 3, \\ 2 & \text { if } 2 m / 3<j \leqslant m-1, \text { or } j=0\end{cases}
$$

$\alpha_{1, j}=1 k$, where

$$
k= \begin{cases}0 & \text { if } 0 \leqslant\left(j+2^{\prime} \quad \bmod m\right)<m / 3 \\ 1 & \text { if } m / 3 \leqslant\left(j+2^{\prime} \quad \bmod m\right)<2 m / 3 \\ 2 & \text { if } 2 m / 3 \leqslant\left(j+2^{\prime} \quad \bmod m\right) \leqslant m-1\end{cases}
$$

and $\alpha_{2, j}=2 k$, where

$$
k= \begin{cases}0 & \text { if } 0<(j+1 \quad \bmod m) \leqslant m / 3 \\ 1 & \text { if } m / 3<(j+1 \quad \bmod m)<2 m / 3, \text { or }(j+1 \quad \bmod m)=0 \\ 2 & \text { if } 2 m / 3 \leqslant(j+1 \quad \bmod m) \leqslant m-1\end{cases}
$$

For each $j=0, \ldots, m-1$, the first components of the $\Sigma$ set $\left\{\alpha_{0, j}, \alpha_{1, j-4^{\prime}}, \alpha_{2, j-2^{\prime}}\right\}$ are $\{0,1,2\}$. Hence, for each $j=0, \ldots, m-1$, the $\Sigma$ set $\left\{\alpha_{0, j}, \alpha_{1, j-4^{\prime}}, \alpha_{2, j-2^{\prime}}\right\}$ is a system of distinct coset representatives for $\langle 01\rangle$, and so the $\Sigma$ condition is satisfied.

Simple computation shows that the set $\left\{-\delta_{0, j},-x_{j-2^{\prime}}-\delta_{1, j-2^{\prime}}, x_{j-1}-\delta_{2, j-1}\right\}$ is equal to $\{00,01,02\}$ if $j \in\{1, \ldots,(m-3) / 3\} \cup\{(m+1) / 2, \ldots,(2 m-3) / 3\},\{02,00,01\}$ if $j \in\{(m+3) / 3, \ldots,(m-1) / 2\} \cup\{(5 m+3) / 6, \ldots, m-1\}$, and $\{02,01,00\}$ if $j \in\{(2 m+$ $3) / 3, \ldots,(5 m-3) / 6\}$. For each of these sets the second components form a permutation of $\{0,1,2\}$, and hence each is a system of distinct coset representatives for $\langle 10\rangle$. As $\alpha_{0, j}, \alpha_{0, j-2^{\prime}}$, and $\alpha_{0, j-1}$ are the same if $j \neq 0, m / 3$ or $2 m / 3$, it follows that the $\Delta$ set $\left\{\alpha_{0, j}-\delta_{0, j}, \alpha_{1, j-2 \prime}-x_{j-2^{\prime}}-\delta_{1, j-2^{\prime}}, \alpha_{2, j-1}+x_{j-1}-\delta_{2, j-1}\right\}$ is a system of distinct coset representatives for $\langle 10\rangle$, if $j \neq 0, m / 3$ or $2 m / 3$. For $j=0, m / 3$ or $2 m / 3$, the $\Delta$ set $\left\{\alpha_{0, j}-\right.$ $\left.\delta_{0, j}, \alpha_{1, j-2^{\prime}}-x_{j-2^{\prime}}-\delta_{1, j-2^{\prime}}, \alpha_{2, j-1}+x_{j-1}-\delta_{2, j-1}\right\}=\{02,11,20\}$ if $j=0,\{00,11,22\}$ if $j=$ $m / 3$, and $\{02,10,21\}$ if $j=2 m / 3$ : as, for each of these $\Delta$ sets the second components form a permutation of $\{0,1,2\}$, each of these $\Delta$ sets is a system of distinct coset representatives for $\langle 10\rangle$. Hence the $\Delta$ condition is satisfied. 
By our construction the $\Theta$ set $\left\{\alpha_{0,0}, \alpha_{0, m / 3}, \alpha_{0,2 m / 3}\right\}=\{02,01,00\}$ and the $\Theta$ set $\left\{\alpha_{2, m-1}, \alpha_{2,(m-3) / 3}, \alpha_{2,(2 m-3) / 3}\right\}=\{21,20,22\}$, the component differences being $\{1,2,0\}$ in both cases. For all other $\Theta$ sets $\left\{\alpha_{i, j}, \alpha_{i, j+m / 3}, \alpha_{i, j+2 m / 3}\right\}=\{i 0, i 1, i 2\}$ in some order; the component differences a re then clearly $\{0,1,2\}$. It follows that each of these $\Theta$ sets is a system of distinct coset representatives for $\langle 11\rangle$. Hence the $\Theta$ condition is satisfied, and $\mathbb{Z}_{3} \times \mathbb{Z}_{3 m}$ admits strong complete mappings by Lemma 11 .

The proof of Lemma 13 yields another construction of a strong complete mapping of $\mathbb{Z}_{3} \times \mathbb{Z}_{27}$

We can now prove the existence of strong complete mappings for noncyclic, abelian 3-groups.

Lemma 14. Every noncyclic, abelian 3-group admits strong complete mappings.

Proof. If $G$ is a noncyclic, abelian 3-group then it is an exercise to show that $G$ admits a subnormal series $\{e\}=K_{1}<\cdots<K_{s}=G$, in which $H_{i}=K_{i} / K_{i-1} \cong G F\left(q_{i}\right)^{+}, q_{i}>3$, if $i<s$, and $H_{s}=K_{s} / K_{s-1} \cong G F(q)^{+}, q>3$, or $\mathbb{Z}_{3 m} \times \mathbb{Z}_{3}, m=3^{n}$. By Lemma 7 , $H_{i}$ admits strong complete mappings for $i<s$. If $H_{s} \cong G F(q)^{+}, q>3$, then $H_{s}$ admits strong complete mappings by Lemma 7 . If $H_{s} \cong \mathbb{Z}_{3 m} \times \mathbb{Z}_{3}$, then $H_{s}$ admits strong complete mappings by Lemma 7 if $m=1$, by Corollary 12 if $m=3$, or by Lemma 13 if $m>3$. Hence, by repeated applications of Lemma $8, G$ admits strong complete mappings.

We are now in a position to characterize finite abelian groups that admit strong complete mappings.

Theorem 15. A finite abelian group admits strong complete mappings if and only if neither its Sylow 2-subgroup nor its Sylow 3-subgroup is nontrivial and cyclic.

Proof. Let $G$ be a finite abelian group and let $p_{1}, \ldots, p_{r}$ be the distinct prime divisors of $|G|$. Let $H_{i}$ be a Sylow $p_{i}$-subgroup of $G$ for $i=1, \ldots, r$. If $p_{i}=2$ for some $i$ and $H_{i}$ is cyclic, then, by Theorem $1, G$ does not admit complete mappings, and hence does not admit strong complete mappings. Similarly If $p_{i}=3$ for some $i$ and $H_{i}$ is cyclic, then, by Theorem 3, $G$ does not admit strong complete mappings.

Next let us assume that the Sylow 2-subgroup of $G$ is trivial or noncyclic and that the Sylow 3-subgroup of $G$ is also trivial or noncyclic. If $p_{i}=2$, then $H_{i}$ admits strong complete mappings by Lemma 9 , if $p_{i}=3$, then $H_{i}$ admits strong complete mappings by Lemma 14 , and if $p_{i} \neq 2$, then $H_{i}$ admits strong complete mappings by Lemma 6 . As $G \cong H_{1} \times \cdots \times H_{r}$, the result follows from repeated applications of Lemma 8 .

\section{Countably Infinite Groups}

In 1950 Bateman [2] proved, using transfinite induction, that any infinite group admits complete mappings. We will adapt Bateman's proof to prove the existence of strong complete mappings for countably infinite groups.

Theorem 16. Any countably infinite group admits strong complete mappings. 
Proof. Let $G=\left\{g_{1}, g_{2}, g_{3}, \ldots\right\}$ be a countably infinite group. Pick $a, b \in G$ and set $x_{1}=a$, $y_{1}=b, w_{1}=a^{-1} b$, and $z_{1}=a b$. Set $X_{1}=\left\{x_{1}\right\}, Y_{1}=\left\{y_{1}\right\}, W_{1}=\left\{w_{1}\right\}$, and $Z_{1}=\left\{z_{1}\right\}$. We will recursively define sets $X_{n}, Y_{n}, W_{n}$, and $Z_{n}, n=1,2,3, \ldots$

Having defined $X_{n}, Y_{n}, W_{n}$, and $Z_{n}$, let $u_{n}$ be the element of $G$ of least index that is not an element of $X_{n} \cap Y_{n} \cap W_{n} \cap Z_{n}$.

If $u_{n} \notin X_{n}$, then set $x_{n+1}=u_{n}$, set $y_{n+1}$ equal to the element of $G$ of least index which is not in $Y_{n}$ and for which $x_{n+1} y_{n+1} \notin Z_{n}$ and $x_{n+1}^{-1} y_{n+1} \notin W_{n}$. Set $z_{n+1}=x_{n+1} y_{n+1}$ and $w_{n+1}=x_{n+1}^{-1} y_{n+1}, X_{n+1}=X_{n} \cup\left\{x_{n+1}\right\}, Y_{n+1}=Y_{n} \cup\left\{y_{n+1}\right\}, W_{n+1}=W_{n} \cup\left\{w_{n+1}\right\}$, and $Z_{n+1}=Z_{n} \cup\left\{z_{n+1}\right\}$.

If $u_{n} \in X_{n}$ but $u_{n} \notin Y_{n}$, then set $y_{n+1}=u_{n}$, set $x_{n+1}$ equal to the element of $G$ of least index which is not in $X_{n}$ and for which $x_{n+1} y_{n+1} \notin Z_{n}$ and $x_{n+1}^{-1} y_{n+1} \notin W_{n}$. Set $z_{n+1}=x_{n+1} y_{n+1}$ and $w_{n+1}=x_{n+1}^{-1} y_{n+1}, X_{n+1}=X_{n} \cup\left\{x_{n+1}\right\}, Y_{n+1}=Y_{n} \cup\left\{y_{n+1}\right\}$, $W_{n+1}=W_{n} \cup\left\{w_{n+1}\right\}$, and $Z_{n+1}=Z_{n} \cup\left\{z_{n+1}\right\}$.

If $u_{n} \in X_{n} \cap Y_{n}$ but $u_{n} \notin W_{n}$, then set $w_{n+1}=u_{n}$, set $x_{n+1}$ equal to the element of $G$ of least index which is not in $X_{n}$ and for which $x_{n+1} w_{n+1} \notin Y_{n}$ and $x_{n+1}^{2} w_{n+1} \notin Z_{n}$. Set $y_{n+1}=x_{n+1} w_{n+1}$ and $z_{n+1}=x_{n+1}^{2} w_{n+1}, X_{n+1}=X_{n} \cup\left\{x_{n+1}\right\}, Y_{n+1}=Y_{n} \cup\left\{y_{n+1}\right\}$, $W_{n+1}=W_{n} \cup\left\{w_{n+1}\right\}$, and $Z_{n+1}=Z_{n} \cup\left\{z_{n+1}\right\}$.

If $u_{n} \in X_{n} \cap Y_{n} \cap W_{n}$ but $u_{n} \notin Z_{n}$, then set $z_{n+1}=u_{n}$, set $x_{n+1}$ equal to the element of $G$ of least index which is not in $X_{n}$ and for which $x_{n+1}^{-1} z_{n+1} \notin Y_{n}$ and $x_{n+1}^{-2} z_{n+1} \notin W_{n}$. Set $y_{n+1}=x_{n+1}^{-1} z_{n+1}$ and $w_{n+1}=x_{n+1}^{-2} z_{n+1}, X_{n+1}=X_{n} \cup\left\{x_{n+1}\right\}, Y_{n+1}=Y_{n} \cup\left\{y_{n+1}\right\}$, $W_{n+1}=W_{n} \cup\left\{w_{n+1}\right\}$, and $Z_{n+1}=Z_{n} \cup\left\{z_{n+1}\right\}$.

If we set $X=\left\{x_{1}, x_{2}, \ldots\right\}, Y=\left\{y_{1}, y_{2}, \ldots\right\}, W=\left\{w_{1}, w_{2}, \ldots\right\}$, and $Z=\left\{z_{1}, z_{2}, \ldots\right\}$, then each element of $G$ appears exactly once in each of the sequences $X, Y, W$, and $Z$. hence, the mapping $x_{n} \mapsto y_{n}$ is a strong complete mapp ing of $G$.

\section{References}

[1] B. A. Anderson. Sequencings and houses. Congr. Numer. 43: 23-43, 1984.

[2] P. T. Bateman. A remark on infinite groups. Amer. Math. Monthly 57: 623-624, 1950.

[3] J. N. Bray. Personal communication.

[4] A. B. Evans. On strong complete mappings. Congr. Numer. 70: 241-248, 1990.

[5] A. B. Evans. The admissibility of sporadic simple groups. J. Algebra 321: 105-116, 2009.

[6] M. Hall, L. J. Paige. Complete mappings of finite groups. Pacific J. Math. 5: 541-549, 1955.

[7] A. Hedayat. A complete solution to the existence and non-existence of Knut Vic designs and orthogonal Knut Vic designs. J. Combin. Theory Ser. A 22(3): 331-337, 1977.

[8] A. Hedayat, W. T. Federer. On the non-existence of Knut Vic designs for all even orders. Ann. Statist. 3: 445-447, 1975. 
[9] J. D. Horton. Orthogonal starters in finite abelian groups. Discrete Math. 79(3): 265-278, 1990.

[10] D. F. Hsu, A. D. Keedwell. Generalized complete mappings, neofields, sequenceable groups and block designs. II. Pacific J. Math. 117(2): 291-312, 1985.

[11] G. O. Michler. Theory of finite simple groups. Cambridge University Press, Cambridge, 2006.

[12] L. J. Paige. A note on finite abelian groups. Bull. Amer. Math. Soc. 53: 590-593, 1947.

[13] Y.-P. Shieh, J. Hsiang, D. F. Hsu. On the existence problems of complete mappings. Preprint.

[14] S. Wilcox. Reduction of the Hall-Paige conjecture to sporadic simple groups. J. Algebra 321: 1407-1428, 2009. 


\section{Corrigendum added 2 October 2018.}

In Theorem 16 it is claimed that any countably infinite group $G$ admits strong complete mappings. Matt Ollis pointed out that this proof fails when $\left\{g^{2} \mid g \in G\right\}$ is finite. The corrected statement of the theorem is:

Theorem 16. If $G$ is a countably infinite group and $\left\{g^{2} \mid g \in G\right\}$ is countably infinite, then $G$ admits strong complete mappings. 